\title{
SOME HIGHLIGHTS OF INTERFEROMETRY IN EARLY RADIO ASTRONOMY
}

\author{
WOODRUFF T. SULLIVAN, III \\ Department of Astronomy FM-20, University of Washington, Seattle, WA \\ 98195 USA
}

\begin{abstract}
Two important episodes in the early development of interferometry in radio astronomy are traced in detail. The first is the use of the sea-cliff interferometer at the Radiophysics Laboratory in Sydney, first by Pawsey for solar observations and later by Bolton for radio star surveys. The second is the development of the Michelson interferometer and the phase switch by Ryle in Cambridge. This also was employed for important observations of the sun and radio stars.
\end{abstract}

\section{INTRODUCTION}

Interferometry has been an essential technique in radio astronomy from the earliest days. I wish to focus on two particular highlights of its development in the years following World War II, and to emphasize the intimate relations between the early radio astronomers, their instruments, and their interpretations of the sky. The highlights are: (1) the invention and exploitation in Australia of the sea-cliff interferometer (1945-48), and (2) the development of the Michelson interferometer at Cambridge (1946-50). These episodes together constituted a large part of the excitement and significance of early radio astronomy. There is not room to continue the story into the origins of aperture synthesis in the early to mid-1950s; for this I recommend articles by Scheuer (1984) and Christiansen (1984). Nor is there space to relate the fascinating story of Robert Hanbury Brown's development of the intensity interferometer at Jodrell Bank in the 1950-53 period (see Hanbury Brown 1974). Astronomy Transformed by Edge and Mulkay (1976) is an excellent source for many of the issues that will be only briefly broached here; furthermore, it provides a detailed analysis of the bitter debates about counts of radio sources in the 1955-65 decade. Other treatments of these debates are given by Mills (1984), Sullivan (1990) and Scheuer (1990). For a compact, but accurate overview of early radio astronomy see Hey (1973).

\section{PAWSEY AND BOLTON IN SYDNEY}

Although the first observations of extraterrestrial radio waves were made in the 1930s by Karl Jansky and Grote Reber (Sullivan 1984b; Reber 1958), 
the major postwar advances in what came to be called radio astronomy took place in England and Australia. The first interferometric observations were made immediately following the war by the young radio physicists who had so successfully developed radar for military applications. In Australia this work both during and after the war was at the CSIR Radiophysics Laboratory in Sydney (Sullivan 1988). Three months after V-J day, in October 1945, Joseph Pawsey (who was to become the leader of Asutralian radio astronomy) and his colleagues Ruby Payne-Scott and Lindsay McCready conducted the first radio interferometry in a program of monitoring solar activity at $200 \mathrm{MHz}$. The climax of their monitoring came in February 1946 when by good fortune the largest sunspot group of the century chose to make its appearance. They now had the opportunity to take advantage of a property of their antenna system (an array of 32 dipoles mounted on a mast) that had previously been more bother than help: a single antenna situated on the edge of a cliff or a hilltop, looking near the horizon over a relatively smooth terrain or over the sea, in fact acts as an interferometer and can achieve far better angular resolution than would otherwise be possible. As the sun rose over the Pacific Ocean, fringes were produced by the interference between the waves travelling directly to the antenna and those that arrived after reflection off the sea (Fig. 1). In classical optics this arrangement is known as "Lloyd's mirror" and the fringes obtained are equivalent to those with a conventional interferometer consisting of the antenna and its imaginary mirror image located under the base of the cliff. The first interferometer in radio astronomy thus involved only one antenna!

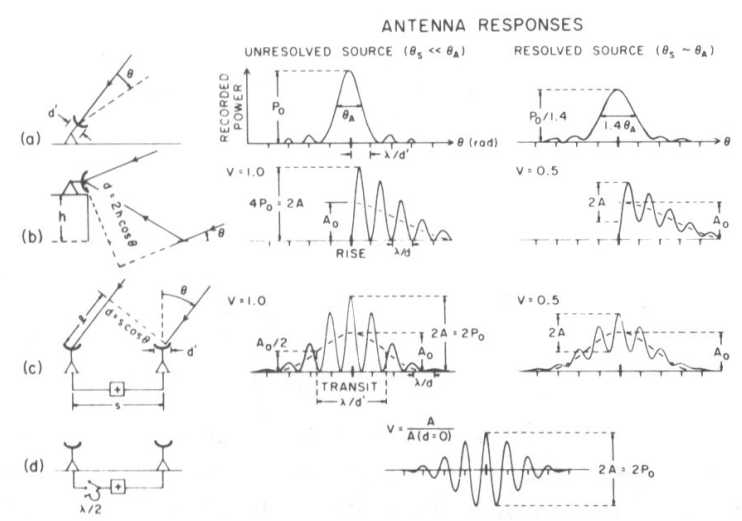

Fig. 1. Antenna responses for (a) a filled aperture, (b) a sea-cliff interferometer, (c) a conventional or Michelson interferometer, and (d) a phase-switched interferometer. On the left are traces of measured power in response to movement across the sky of an unresolved source; on the right from a resolved source of the same flux density. Tick marks on all the abscissae are at intervals of $\lambda / d$ and received powers are all in terms of that from an unresolved source for a single antenna. For the interferometers, the visibility $\mathrm{V}$ of the fringes is shown.

With antennas about $100 \mathrm{~m}$ above the sea, the fringe lobes were spaced by about 25', much better than the 6-degree beam of the antenna itself. It is important to note that this phenomenon was nothing new to those who 
had been developing radar systems, for during the war radar beams often pointed near the horizon, as with search radars on a ship or a coastline. In such a situation the reflected signal from a distant aircraft was well known to oscillate as it passed through the radar's lobes. This effect was both a blessing and a curse to the radar systems designer, for it could be used to gather precise information on a target's height, but on the other hand it meant that lowflying aircraft could sneak in "under" a radar, since the first lobe was not at the horizon, but above it by a considerable amount, especially if the antenna was not high above its surroundings.

So Pawsey and his colleagues used this sea-cliff interferometer [1] to advantage as the bespotted sun rose over the Pacific. They observed not only an enhanced general level of solar emission, but also occasional strong bursts that produced striking, superimposed oscillations, the interferometric fringes (Fig. 2). The very presence of these oscillations implied that the source of the solar signal was a good bit smaller than the 30' size of the optical sun. Exactly how much smaller the emitting region was, as well as its location, could of course be worked out through details of the fringes' amplitudes and phases. In fact they found that the emitting region had a width of 8-13' (implying brightness temperatures as high as $310^{9} \mathrm{~K}$ ) and on any given day coincided with the giant sunspot group being carried along by the sun's rotation. This was the first demonstration of a positional association between sunspots and radio bursts.

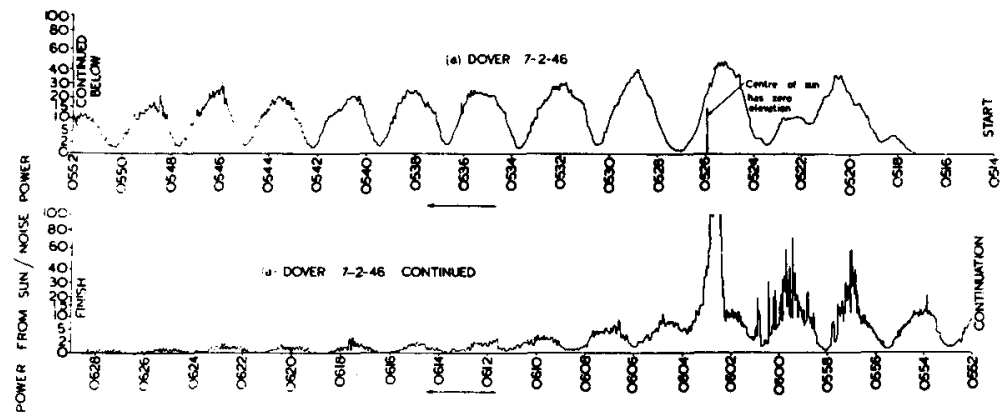

Fig. 2. Sea-cliff interference patterns obtained at $200 \mathrm{MHz}$ in February 1946. When the sun rose, the fringes suddenly appeared (note that the "radio sunrise" came earlier than the optical sunrise) and then gradually faded away about an hour later as the sun moved above the beam of the antenna. The very fast variations and intense signals recorded at 0600 represent a solar outburst. (McCready, Pawsey and Payne-Scott 1947)

In a seminal paper submitted to the Proceedings of the Royal Society in July 1946, McCready, Pawsey and Payne-Scott (1947) reported the above results and also explained many basics of the sea-cliff interferometer, considering effects such as refraction (the worst uncertainty), the earth's curvature, tides, and imperfect reflection from a choppy sea. As mentioned above, many of these effects had already been worked out before the war. But Pawsey's group also introduced a vital new principle, namely that their interferometer was sensitive to a single Fourier component (in spatial 
frequency) of the brightness distribution across the sun, and that in principle a complete Fourier synthesis could be achieved if one had enough observations with interferometers of different effective baselines. They wrote:

Since an indefinite number of distributions have identical Fourier components at one [spatial] frequency, measurement of the phase and amplitude of the variation of intensity at one place at dawn cannot in general be used to determine the distribution over the sun without further information. It is possible in principle to determine the actual form of the distribution in a complex case by Fourier synthesis using information derived from a large number of components. In the interference method suggested here...different Fourier components may be obtained by varying the cliff height $h$ or the wave-length $\lambda$. Variation of $\lambda$ is inadvisable, as over the necessary wide range the distribution of radiation may be a function of $\lambda$. Variation of $h$ would be feasible but clumsy. A different interference method may be more practicable.

Much of the subsequent technical development of radio astronomy was to be concerned with this method of making high-resolution cuts across sources, and eventually complete maps; it is of course still the basis of this conference forty-five years later. But the last two sentences of the above quotation were prophetic, for it was not sea-cliff interferometry, but the more tractable and flexible conventional interferometry with two separate antennas (see below), that made source mapping a reality in the $1950 \mathrm{~s}$.

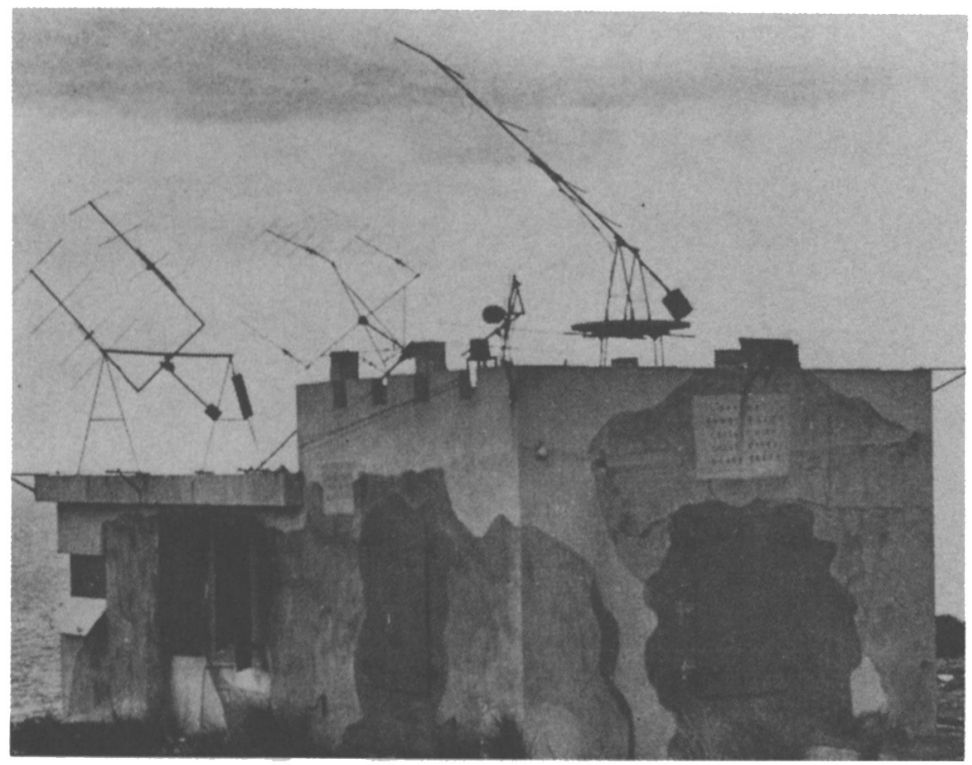

Fig. 3. Yagi antennas for 60,100 , and $200 \mathrm{MHz}$ used for monitoring the sun and measuring its circular polarization at Dover Heights, Sydney (1947). With the Yagi elements parallel and pointing toward the horizon, John Bolton and his group made their first studies and surveys of discrete sources. (courtesy CSIRO Division of Radiophysics) 
John Bolton, a Radiophysics staff member who had been a radar officer in the Royal Navy, continued with sea-cliff interferometry of radio sources until as late as 1952 (Bolton 1982). Together with his engineer colleague Gordon Stanley, he first studied the enigmatic discrete source in Cygnus discovered in England by J.S. Hey's group (Hey, Parsons, and Phillips 1946). In mid-1947 with a system that was nothing more than a pair of $100 \mathrm{MHz}$ Yagis (similar to Fig. 3) connected to a converted radar receiver, they surveyed the southern sky over three weeks and reliably found the Cygnus source, as well as hints of two weaker ones.[2] They spent several months checking out the Cygnus source, and by the end of the year submitted papers to Nature and the very first issue of the Australian Journal of Scientific Research (Bolton and Stanley 1948a,b). Cygnus usually gave a workably strong set of fringes as it rose (Fig. 4), and this directly implied that the radiation emanated from a very small, single region of the sky. But the source never rose more than 15 degrees above the northern Sydney horizon and observations were continually harassed by the strong intensity fluctuations that had led to Hey's discovery in the first place.
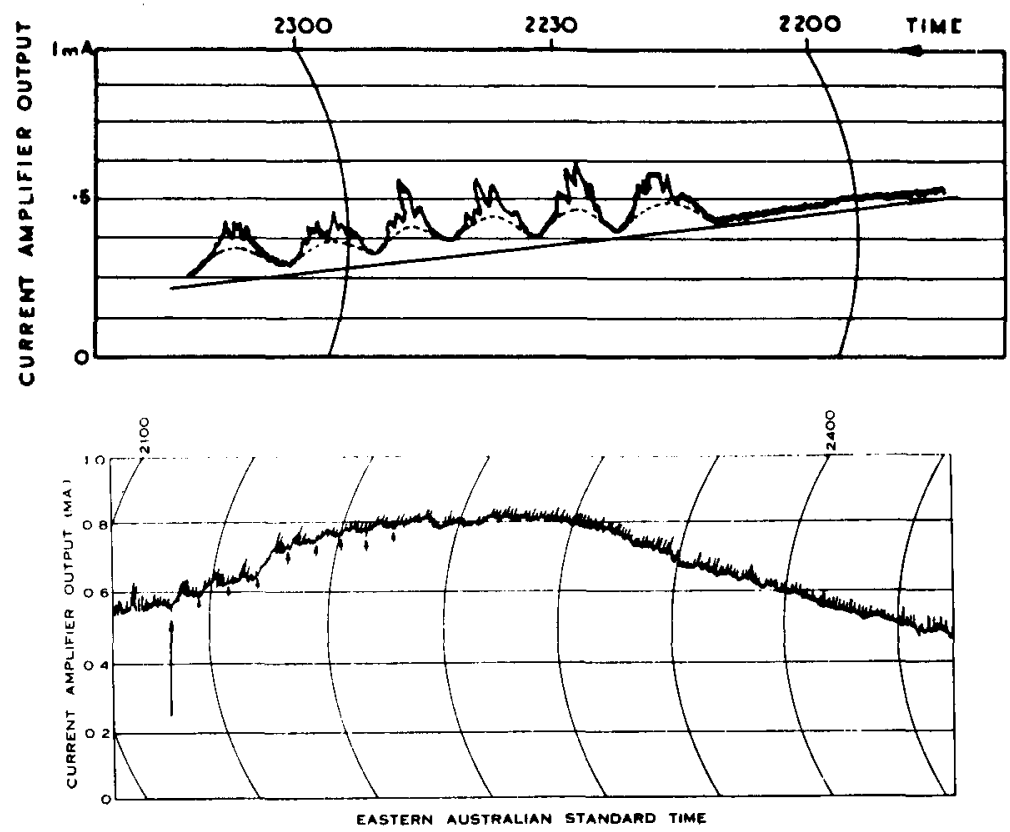

Fig. 4. Sea-cliff interference patterns obtained at $100 \mathrm{MHz}$ at Dover Heights, Sydney, for Cygnus A in June 1947 (top) and for Taurus A in November 1947 (bottom, discovery recording). Note the ionospheric scintillations superimposed on the Cygnus fringes and the sloping baselines from galactic background radiation. For the Taurus A record, the rising point and probable minima of the weak fringes are shown by arrows. Vertical lines on this record are due to interference from a timing mechanism. (Bolton and Stanley 1948b, 1949) 
The heart of their study was concerned with the size and position of the source. Size came from the technique worked out on the sun over a year before, namely the ratio of the intensity of fringe maximum to minimum. But Cygnus gave difficulties with (1) subtracting off a considerable baseline slope caused by strong galactic noise in the vicinity, (2) isolating the constant component from the variable, and (3) determining a proper upper limit for the fringe minimum (Fig. 4). They estimated that the maximum-to-minimum ratio was at least 50 , implying that the source size was less than $8^{\prime}$ (about one-eighth of the lobe separation). Hey had inferred that the Cygnus fluctuations must arise from a discrete source or collection of sources, perhaps scattered over several degrees of sky, but here was strong evidence for a single, small source.

With a source size in hand, they moved on to the even trickier task of a position. This involved measurement and interpretation of the timing and spacing of fringes in terms of sky geometry and radio wave propagation. One had to find the sidereal time when the source was highest in the sky (culmination) and the length of the arc travelled by the source between rising and culmination. But Bolton and Stanley were forced to tie together observations at three different cliffs around Sydney in order to secure reliable data; furthermore, the necessary corrections for refraction were large and, as it turned out, uncertain. In the end, the derived position was quoted good to 7'. With this first well-defined position for the Cygnus source (Hey's group had been able to give its position only to within 5 degrees!), their next step was of course to consult optical catalogs and photographs. But this was disappointing, for they found no optical counterparts and stated that "the radio noise received from this region is out of all proportion to the optical radiation." They did, however, request Richard Woolley, the director at Mt. Stromlo, to take a special photograph of that portion of the sky, and this appeared as a plate in their paper, along with a tracing-paper overlay indicating their source position and error box. It certainly appeared a nondescript patch of sky.[3]

Given that there was no optical counterpart, could one nevertheless put any constraints on the distance to the object? Since they had been observing the source for three months, the changing position of the orbiting earth might have caused an apparent shift in position if the object were nearby. But they had detected no shift greater than 2.5' (corresponding to their $10 \mathrm{sec}$ accuracy in timing the sudden appearance of the source at rising), and this meant the source was at least ten times the 50 light-hour distance to Pluto, that is, well outside the solar system. But how far? Bolton and Stanley could only suggest that the farthest imaginable would be if somehow the radio object were a star with total power output similar to that of the sun, but all channeled into the radio spectrum. That distance worked out to be 3000 light years.

Just as Bolton and Stanley were writing up these results, they received an interesting communication from Pawsey, who was then on the first leg of an around-the-world tour. He had visited Mt. Wilson Observatory in Pasadena and there found Rudolph Minkowski "intensely interested" in the Cygnus results and willing to undertake observations directed toward finding an optical counterpart. Pawsey's letter ended with a raft of suggestions from Minkowski for possible places to look for radio noise: 
The Magellanic Clouds [are] the nearest external galaxies, abnormal with much dust and blue stars....If we are interested in interstellar dust, etc. the "Crab Nebula", NGC 1952, is a good sample. If white dwarfs are of interest, the companion of Sirius is a convenient sample. The Orion region is a region of emission nebulae. [But] I do not think these ideas get us very far. I should recommend the method of empirical searching; our tools are not too fine to prevent this.[4]

With the Cygnus case temporarily closed, Bolton and Stanley indeed set out in November 1947 to search the sky in Pawsey's "empirical" fashion. Stanley had made significant improvements to their receiver's short-term stability, in particular through constructing power supplies able to provide voltages stable to a part in a few thousand. Very weak fringes could now be reliably detected. They methodically took records at different points along the eastern horizon, and indeed fringes for several sources appeare $d$ and were confirmed in the next few months. As it became clear that the sky had a lot more to offer than the Cygnus source, Bolton introduced the familiar nomenclature that the strongest source in a constellation would be called $A$, the next B, etc. And so their second source became Taurus A, one-sixth as strong as Cygnus $\mathrm{A}$, followed by Coma Berenices $\mathrm{A}$ at a similar level. The uncertainties of this work can be appreciated by noting that Taurus A appeared nicely on one November night (Fig. 4), but it took another three months for confirmation of its existence and measurement of a position good enough for the assigning of a constellation.

By February 1948 Bolton had surveyed about half of the southern sky (manmade interference made daytime observations nearly worthless) and had good cases for six new discrete sources. He wrote up a short note to Nature (Bolton 1948) announcing that a new class of astronomical object existed: Cygnus was not unique, either in its existence or in its lack of association with "outstanding stellar objects." Upper limits on the new sources' sizes were no better than 15-60', but Bolton was becoming convinced that all these discrete sources were truly stellar, "distinct 'radio-types' for which a place might have to be found in the sequence of stellar evolution." Since even the most powerful solar-style bursts would not do the trick, he appealed to either pre-main sequence, collapsing, cool objects or to old, hot objects related to planetary nebulae. [5]

After this survey Bolton chose to improve his source positions, in particular to eliminate systematic errors, by observing source setting as well as rising. High westward- and northward-facing cliffs were needed and so Bolton and Stanley headed off to New Zealand in the southern winter of 1948. The superior observations in New Zealand put an even tighter limit on Cygnus A's size $\left(<1.5^{\prime}\right)$, and, together with simultaneous observations by Bruce Slee in Sydney, provided strong evidence that most of the intensity fluctuations originated in the earth's atmosphere, not in the source itself. It also became apparent that incorrect refraction corrections and other problems had thrown most previous positions 5 to 10 degrees off. Some even changed names as when Coma Berenices A migrated into Virgo. But Cygnus A was still vexing, as neither its new position (shifted about one degree south from earlier) nor its old one agreed with that measured by Martin Ryle at Cambridge. For a while it seemed that the source might actually be moving, but after about six months 
of sorting out, both Hemispheres admitted earlier errors and were able to agree on a common position.

The really beautiful outcome of the new positions of $\sim 10^{\prime}$ accuracy was that for the first time optical counterparts could indeed be tentatively suggested (Bolton, Stanley and Slee 1949). And these were no ordinary objects. Taurus A was associated with the Crab nebula, the expanding shell of a supernova known to have exploded 900 years before (Bolton and Stanley 1949); Centaurus A was found to coincide well with one of the brightest and strangest nebulosities in the sky, so peculiar that astronomers were not even sure whether or not it was part of our Galaxy; and Virgo A's position agreed with that of a bright elliptical galaxy five million light years away. These associations quickened interest in the study of discrete sources and caused several optical astronomers, among them Minkowski, to take serious note. But here we must leave the Australian story and switch to the Northern Hemisphere.

\section{RYLE AT THE CAVENDISH}

Martin Ryle and his colleague Derek Vonberg had both worked on radar development at the Telecommunications Research Establishment during World War II and then returned to Cambridge University to pursue Ph.D.'s under the direction of the ionospheric radio physicist J.A. "Jack" Ratcliffe. Most of their first year in the Cavendish Laboratory was spent in restocking bare shelves with cheap and abundantly available surplus radar equipment, as well as testing various schemes to check out the "solar noise" phenomenon. But sometime in the winter of 1945-6 Ryle came up with the idea of an interferometer, two separated small antennas whose signals would be combined by cable. The fringes from such a setup would provide excellent angular resolution, and by increasing the spacing to the point where the fringe amplitudes became weaker, one could estimate the radio source's size (Fig. 1). Furthermore, since the beam of each individual element was broad, a source such as the sun could be monitored for several hours each day without any need for tracking. This scheme was of course analogous to the optical interferometer used by Albert Michelson and Francis Pease in the 1920s for measuring the angular diameters of stars. T.L. Eckersley (1938) had even employed such an instrument for ionospheric research before the war, but Ryle $(1976: 4 \mathrm{~T})[6]$ recalled that the idea came to him from elsewhere:

I don't think the idea arose by analogy with an optical Michelson interferometer because one had forgotten all one's physics by the end of the war. I think it arose from the idea that if you have a null by interference between two aerials, then this could fairly easily tell you something about a compact source. And then you could make that null narrower by separating the aerials. I suppose we were reinventing the Michelson interferometer, but I think it came from a rather simpleminded thinking about aerials rather than from saying, "Ah, I remember in my physics book - optics."

The $175 \mathrm{MHz}$ receiver system was also novel, designed by Ryle to allow a continuous, accurate measurement no matter how noise power level might 
vary (such as during a solar burst, or during fringe oscillations). They called it the "cosmic radio pyrometer," but even today it is known as a Ryle-Vonberg receiver (Ryle and Vonberg 1948). The antenna signal was compared (at a rate of $23 \mathrm{~Hz}$, using a capacitance switch from a German Junkers 88 nightfighter) with a reference noise source, whose magnitude was then continually varied so as to equal the signal. The noise power emitted by this reference was thus a measure of the signal strength, and, so long as the reference source could track gain changes accurately and quickly enough, omnipresent variations in gain or other receiver parameters, for instance those arising from a varying mains voltage, had minimal effect.
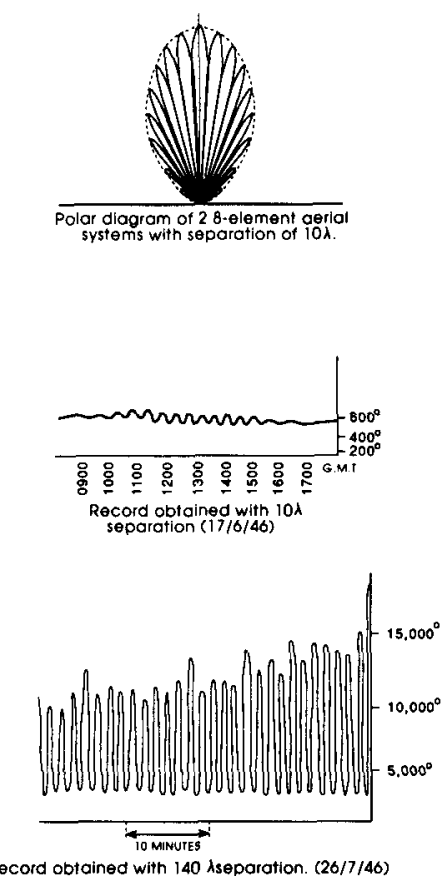

Fig. 5. Calculated antenna response and observed fringes at $175 \mathrm{MHz}$ from an active solar region in July 1946. These observations are the first with a Michelson interferometer and the Ryle-Vonberg receiver, but not yet with a phase-switch (note the large offsets). (Ryle and Vonberg 1946)

Each interferometer element was an array of eight halfwave dipoles, mounted on a support of angle iron covered with a chicken-wire reflecting screen. They were connected by low-loss, air-spaced coaxial cable whose nickname of "Gerry cable" revealed its origin. Ryle had managed to scrounge a kilometer of this stuff - nothing of comparable quality was available in Britain for many years - but it came only in relatively short lengths. This necessitated tedious hours making plug and socket connectors, which unhappily led to severe attenuation whenever rainwater leaked in. These cables and their troublesome connectors were used by the group for almost a decade. 
By June 1946 they had a going system, but interest in the Milky Way, which gave the same trace every day, quickly waned in favor of the changeable sun. In late July a giant sunspot group appeared and Ryle and Vonberg (1946) seized the opportunity to attempt a measurement of the angular size of the accompanying radio-emitting region. Within a week they hauled their dipole array to three ever-more-distant spacings, from 25 to 140 wavelengths $(240 \mathrm{~m})$, necessitating an invasion of the adjacent University rugby ground. The trouble was worth it, for the ratio of maximum to minimum intensity measured on the longest baseline, at which the fringes were spaced by $25^{\prime}$ (Fig. 5), enabled them to place an upper limit of 10' on the emitting region's east-west extent and therefore a lower limit on its brightness temperature of $210^{9} \mathrm{~K}$.

By crossing the polarity of the two elements, they also measured 100argue that the strong magnetic field of the sunspot group was important for the radio emission process. We can now see that this episode was not only an important step in solar astrophysics, but also constituted Martin Ryle's first step in a thirty-year journey of developing new techniques for interferometry.

During the early months of 1948 Ryle became interested in Hey's discrete source of emission in Cygnus, as well as in Bolton's many additional "radio stars." The solar records taken over the course of 1947 had often shown weak ripples that were tantalizing indications of possible discrete sources, but of inconsistent strength from day to day. Ryle decided to investigate the state of polarization of the radiation from the Cygnus source, in order to see whether it corresponded to the circularly polarized sunspot radiation or to the unpolarized quiet sun.

Ryle and Graham Smith, who had recently joined the group as a research student, first tried to observe Cygnus simply by tilting the solar dipole-array antennas up to the appropriate declination. But their $80 \mathrm{MHz}$ interf erometer gave only weak, unusable signals and the $175 \mathrm{MHz}$ interferometer none at all. So they increased the antennas' gains by converting them into four-Yagi arrays and moved the spacing to 120 wavelengths in order to minimize interference from general galactic emission. Now they hit the jackpot, for on the first night in which they left this new $80 \mathrm{MHz}$ system running (still with the noise-diode balancing scheme), not only did fringes from the Cygnus source show up, but three hours later even stronger ones - they had discovered Cassiopeia A, the strongest radio source in the sky:

We said, "Let's build an instrument to look for [this fluctuating region in Cygnus]." And it was actually very exciting because we built this instrument and left it running and overnight, damn it, there were two of them! (Ryle 1976:17T)

I remember turning up at the laboratory after the thing had been set running, and there was an interferometer trace of Cygnus just as it should be - absolutely marvellous. And then three hours later, another [sinusoidal] trace, which subsequently turned out to be Cassiopeia A...with a different periodicity than Cygnus $\mathrm{A}$. This was a complete surprise. We thought it must be something to do with the way things moved in the sky, but neither of us had any training in celestial coordinates....I went away to think about it, and I decided the fringe 
rates would be different according to the distance from the pole. So that was my first introduction to spherical astronomy.... remember then coming into the Cavendish Lab an hour or two later and saying to Ryle and Ratcliffe, "I think it's in a constellation called Cassiopeia." (Smith 1976:10-11T)

Ryle and Smith (1948) had accidentally discovered not only a new radio star, but a powerful method for determining positions. The right ascension came from measuring the sidereal time when the source transited, that is, when the fringes reached greatest amplitude, and the declination from the periodicity of the fringes. Although their individual antennas had $a \sim 60$-degree beam and therefore very little discrimination in the north-south direction, the fact that stars nearer the celestial pole moved more slowly across the interferometer's pattern of lobes could be used as a neat measure of declination.

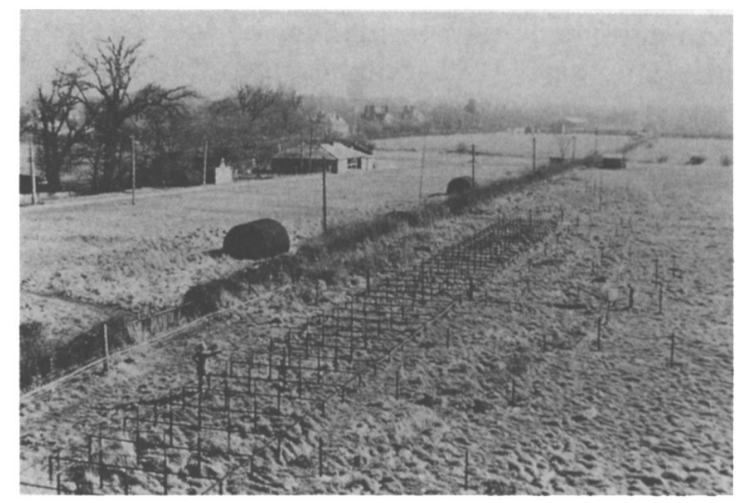

Fig. 6. The eastern element of the Long Michelson (1C) interferometer (ca 1950), with Graham Smith standing alongside. The element consists of an array of $2 \times 4081 \mathrm{MHz}$ half-wave dipoles mounted 1/4 wavelength above a reflecting screen. (courtesy Bruce Elsmore)

Enticed by these strong sources and hints of many weaker ones, Ryle decided that the next step was to find more of these radio stars by building an interferometer with far greater sensitivity. Thus was born what the group called the "Long Michelson" (Fig. 6).

Ryle shared strongly in the Cavendish tradition of simple apparatus applied to experiments of elegant design, and to him the east-west Michelson interferometer was a particularly economical device: its small, stationary antennas allowed one to neatly derive both coordinates for all the radio stars in the sky, and in only one day. Element spacing remained at about one hundred wavelengths (of $3.7 \mathrm{~m}$ each), but each element was now a $10 \times 1$ wavelength broadside array of 40 half-wave dipoles made from brass tubing. This was designed to be more sensitive and to produce fewer fringes for each passage of a radio star through the primary beam of the antennas, thus allowing the "essential maximum," that is, the fringe of greatest amplitude, to be recognized. Its 175 pound $(\$ 500)$ cost made it by far their largest project. Observations began in December 1948 and within a few months Ryle, Smith 
and Bruce Elsmore, who had joined the group as a technician, had catalogued a couple of dozen sources. In principle the chart record from just a single twenty-four-hour run was sufficient for a complete survey of the northern sky, but in practice it was found that the fringe patterns were inconstant from day to day (Fig. 7). The weaker sources were especially bothersome - sometimes there, sometimes not.

These first observations with the Long Michelson were almost too successful: the sky was apparently so full of fainter sources that their fringe patterns on the charts often blended, making it difficult to tell how many sources were present and where they were located. In April 1949 Ryle gave a paper to the Royal Astronomical Society about his survey results, showing a map with 21 new sources besides Cas A and Cyg A. But he was not at all confident of their positions, for he noted that "the distribution on the map is...not directly related to that actually occurring in the sky as the detection of a source on the record is often limited by the confusion arising from adjacent sources." [7] He thus decided to double the east-west length of each array, so that sources now exhibited only about five fringes on their daily transit, not ten, and signals were yet stronger (Fig. 7). More importantly, he came up with an ingenious solution to difficulties stemming from the strong galactic background radiation. Because one was constrained to set the amplifier gain on the recorder such that the trace always stayed on the chart, sources could not be measured if they registered less than a few percent of the maximum background signal recorded over the entire day. Ryle's fix came to be known as the phase switch, and its merit was such that it soon became the standard everywhere; for example, Bernard Mills (1952) installed it at the Radiophysics Laboratory for his first survey of radio sources in 1950.[8]

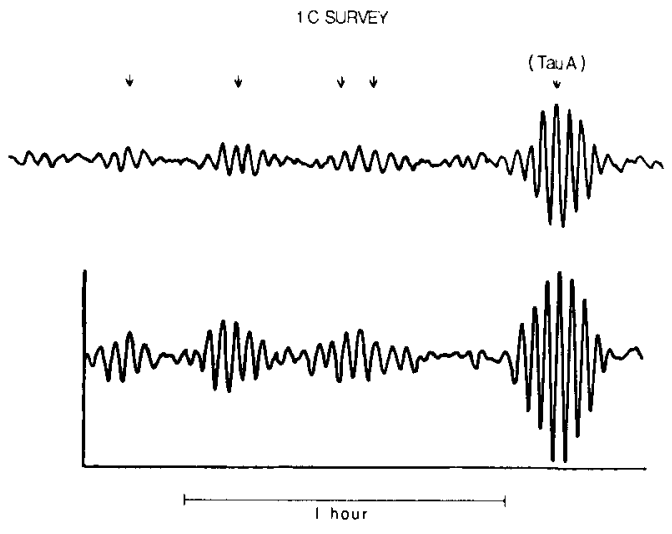

Fig. 7. Traces of the same portion of sky on two different days of observation with the "Long Michelson" interferometer (Fig. 6) for the $1 \mathrm{C}$ survey in 1949-50. The bottom trace comes from Ryle, Smith and Elsmore (1950) and the top from Smith's 1951 Ph.D. thesis; the positions of listed $1 \mathrm{C}$ sources are indicated with arrows. 
Ryle originally invented the phase-switch in connection with advisory work on underwater sound detection that he was then doing for the Admiralty - for a while the group called it the "mermaid hunter." In the underwater warfare case, one was trying to pick out the signal of a submarine against the background noise caused by fish, currents and other vessels, but the principles were identical for detecting a radio star against the Milky Way background. Ryle laid out details of the technique in an October 1947 report,[9] but over a year passed before the phase switch was used at Cambridge.[10] The reason for this delay is not known, but Ryle's student Kenneth Machin (1981:134B:570) vividly recollected:

Martin came in one day and said "Hey, I've just had a fantastic idea!" So he got out a piece of paper and drew on it on the floor. "Right," he said, "it's eleven o'clock, shall we go and try it?" And so we modified a standard set in an hour and a half - we simply rewired the RF switching so as to put an alternative half-wave in. And that showed it worked.

The phase switch was a capacitor switch rapidly switching to a configuration that inserted an extra half-wavelength of cable into one arm of the interferometer. This phase reversal of course caused the lobes of the interferometer's antenna pattern to oscillate between two sky positions exactly one-half lobe spacing apart. And when one now synchronously recorded the difference of the signal strength in the two switch positions, any radiation coming from a region of sky larger than the size of a lobe spacing would be cancelled out, and the only remaining signals would be from discrete sources (Fig. 1). Furthermore, it turned out that this technique not only eliminated the deleterious effects of the galactic background (for baselines longer than one hundred wavelengths), but also minimized those from deficiencies such as manmade interference, receiver gain variations, and inequalities between arms of the interferometer. Ryle's (1952) tutorial paper on the phaseswitched Michelson interferometer (and its superiority over both the seacliff interferometer and dish antennas) became the essential primer for all his students and is still today a paper worth reading.

By mid-1950 the Long Michelson had spawned a list of 50 radio stars (later to be known as the $1 \mathrm{C}$ catalog) with quoted positional accuracies typically of 1 to 3 degrees (Ryle, Smith and Elsmore 1950). The group had also brought forward a series of arguments that these radio stars represented a "hitherto unobserved type of stellar body, distributed widely throughout the galaxy," and that the galactic background radiation arose from the integrated effect of these new stars. These stars were numerous $\left(\sim 3 \mathrm{pc}^{-3)}\right.$, had a cool photosphere , and had a hot corona that produced cosmic rays as well as radio emission. Repeated observations of the stronger sources with the Long Michelson revealed no effects of annual parallax, proper motion, or long-term intensity changes. But there is no space to pursue further the story of the radio stars; for more details see Edge and Mulkay (1976) and Sullivan (1990).

It is important to stress Ratcliffe's influence on early interferometry through his teaching regarding the physical applications of Fourier transforms. Many of my interviewees have recalled the lucidity, insightfulness, breadth, and long-term influence of his series of about thirteen lectures on physical applications of Fourier transforms; [11] Ratcliffe has even been rated as the 
finest lecturer at the Cavendish in this century. This influence was not only on Ryle and his group, but also on Pawsey (who studied under Ratcliffe at the Cavendish in the early 1930s) and other Australians. A case in point is Ronald Bracewell, who earned his Ph.D. at the Cavendish in the late 1940s, returned to Sydney, and himself made important contributions to Fourier theory for antennas and aperture synthesis. Bracewell (1980:131A) recalls how Ratcliffe never would simply state something like "the Fourier transform of a cosine is a (double) delta function," but rather "a cosine variation in the ionosphere's density will cause an incident plane wave to be detected at the ground as two plane waves coming from either side of the vertical." It was etched upon Ratcliffe's students' minds that a measurement of the pattern of signal phase and amplitude on the ground allowed one to map the sky brightness distribution and/or properties of any intervening medium. This approach was particularly profitable for Ryle's work on principles of interferometry,[12] as well as Antony Hewish's (also under Ratcliffe's direction) on scattering and scintillation of radio waves by the ionosphere.

Ryle's researches were also strongly supported by the Cavendish Professor, William Lawrence Bragg, who in 1915 had received (at age 25) the Nobel Prize with his father for pioneering the technique of X-ray crystallography. Its application at the Cavendish to biological molecules by F.H.C. Crick and J.D. Watson culminated in 1953 in one of the fundamental discoveries of twentieth century biology: the unravelling of the double helix structure of deoxyribonucleic acid. X-ray crystallography involves measuring the diffraction pattern of X-rays after their passage through a lattice-like arrangement of molecules. The Fourier transform of this pattern then yields the sought-for molecular structure. The technique is therefore directly analogous to the radio astronomer's analysis of extraterrestrial radio waves, and Bragg was excited and delighted by this similarity despite the disparity in scales: a shift in wavelength of $\sim 10^{10}$ and in structure sizes of $\sim 10^{31}$ ! ।

\section{CLOSING}

I hope that these details of some of the early developments in interferometry and the accompanying radio observations have given the reader an appreciation for the fundamental contributions of people like Pawsey, Bolton, Ratcliffe, Ryle, and their colleagues. Although science in that era differed in many ways from that of the 1990 s, we should never forget that we are the heirs of the intellectual fortune they amassed.

\section{ACKNOWLEDGMENTS}

This paper is primarily drawn from portions of two chapters of my almostfinished monograph on the early history of radio astronomy. I thank the many interviewees (some of whom were at this meeting), librarians, and archivists who have aided my project over the years. In particular, the archives of the CSIRO Radiophysics Division and of Martin Ryle at Churchill College have been indispensible for the researches leading to this paper. I am also grateful to the NSF Program in History and Philosophy of Science for substantial 
support over the years to this project. Finally, I commend my editors Tim Cornwell and Rick Perley for their patience in extracting these long-overdue pages.

\section{NOTES}

[1] I use the term "sea-cliff interferometer," although at the time the arrangement was called either a "sea interferometer" or a "cliff interferometer." [2] Bolton, "Summary of Cygnus Results - 4th-25th June, 1947," 1 page, undated, file A1/3/1a, CSIRO Radiophysics Division archives, Sydney (= RPS). Details in Bolton (1982) have also been used in this section.

[3] As it turned out, Bolton and Stanley's first Cyg A position was a full degree north of the correct position and so there was no chance of finding an optical counterpart, although note that even positions measured years later to accuracies of a few arcminutes were unable to disclose an optical identification. [4] Pawsey:E.G. Bowen and Bolton, 11 November 1947 (from Lincoln, Nebraska), file A1/3/1a, RPS.

[5] It was only fitting that an aleing Australian star should radiate its Swan song in the form of Cygnus-type radio noise.

[6] Citations of the form Jones (1975:23T) refer to p. 23 of the transcript of my 1975 interview with Jones; Jones $(1975: 87$ B:300) refers to Tape 87, Side B, position 300 of Jones's (untranscribed) interview.

[7] M. Ryle, "Discrete celestial sources of radio-frequency radiation," presented to the RAS on 8 April 1949, as recorded in Observatory 69, 85-7 (1949).

[8] Mills learned of the phase-switch idea from Robert W.E. McNicol, who returned to ionospheric research in Brisbane after receiving his $\mathrm{Ph} . \mathrm{D}$. under Ratcliffe in 1949. Pawsey was careful, however, to insure that Mills gave Ryle priority for the technique. Also see Mills (1984). [Pawsey:Ryle, 1 May 1951, box 1/2, Ryle papers, Churchill College, Cambridge University (= RYL); F.F. Gardner:author, 12 November 1986]

[9] Ryle, "The detection of a small signal originating from a point source against a large background signal of diffuse origin," 29 October 1947, 8 pp., file 12 (uncat.), RYL. This report describes phase-switching in some detail, but only gives hints now and then that the intended application deals with underwater naval operations (for example, the use once of the term transducer).

[10] The date of introduction of phase-switching is uncertain. The written record of the RAS talk of April 1949 (note 7) suggests that the data discussed were not taken with phase-switching. In a letter of 7 June 1949 to Appleton [box 1/1, RYL], Ryle mentioned experiments with a "new system for obtaining better discrimination against the galactic background." This would seem to imply that the technique was introduced in April or May 1949, but another document ("Solar noise programme," [file 6 (uncat.), RYL]) suggests that phase-switching went into operation as early as its date of February 1949. [11] Ratcliffe's lectures on Fourier transforms were never published as such, but stenciled notes entitled "Fourier analysis and correlation functions in diffraction and circuit theory: a physical approach" were made in 1956. His most pertinent publication was "Some aspects of diffraction theory and their application to the ionosphere," Rept. Prog. Phys. 19, 188-267 (1956). 
[12] The first statement found in Ryle's archives of the principles of Fourier mapping of the sky dates from as early as 1946: "If a maximum/minimum ratio [of the fringe amplitudes from the sun] were obtained with a large number of equivalent aerial spacings, it should be possible, by carrying out a harmonic analysis, to derive a true line distribution of power intensity, and this would show up the contribution of multiple spots and the disc as a whole." [Ryle, "Notes on future noise programmes," 23 August 1946, 2 pp., file 18 (uncat.), RYL]

\section{REFERENCES}

Note that an asterisk indicates that a paper is reprinted with commentary in Classics in Radio Astronomy (1982), W. T. Sullivan, III (Ed.), Dordrecht: Reidel.

Bolton, J. G. (1948). "Discrete sources of galactic radio frequency noise." Nature 162: 141-2.

Bolton, J. G. and Stanley, G. J. (1948a).* "Variable source of radio frequency radiation in the constellation of Cygnus." Nature 161: 312-3.

Bolton, J. G. and Stanley, G. J. (1948b). "Observations on the variable source of cosmic radio frequency radiation in the constellation of Cygnus." Austral. J. Sci. Res. A1: 58-69.

Bolton, J. G. and Stanley, G. J. (1949). "The position and probable identification of the source of galactic radio-frequency radiation TaurusA." Austral. J. Sci. Res. A2: 139-48.

Bolton, J. G., Stanley, G. J. and Slee, O. B. (1949).* "Positions of three discrete sources of galactic radio-frequency radiation." Nature 164: 1012.

Bolton, J. G. (1982). "Radio astronomy at Dover Heights." Proc. Astron. Soc. Australia 4: 349-58.

Christiansen, W. N. (1984). "The first decade of solar radio astronomy in Australia." pp. 113-31 in Sullivan (1984a).

Eckersley, T. L. (1938). "A wireless interferometer." Nature 141: 369-70.

Edge, D. O. and Mulkay, M. J. (1976). Astronomy Transformed: The Emergence of Radio Astronomy in Britain. New York: John Wiley and Sons.

Hanbury Brown, R. (1974). The Intensity Interferometer. London: Taylor and Francis.

Hey, J. S., Parsons, S. J. and Phillips, J. W. (1946).* "Fluctuations in cosmic radiation at radio-frequencies." Nature 158: 234.

Hey, J. S. (1973). The Evolution of Radio Astronomy. London: Elek Science.

McCready, L. L., Pawsey, J. L. and Payne-Scott, R. (1947).* "Solar radiation at radio frequencies and its relation to sunspots." Proc. Roy. Soc. A190: $357-75$.

Mills, B. Y. (1952). "The distribution of the discrete sources of cosmic radio radiation." Austral. J. Sci. Res. A5: 266-87. Erratum: Austral. J. Physics, Vol. 6, p. 125 (1953).

Mills, B. Y. (1984). "Radio sources and the $\log N-\log S$ controversy." pp. $147-65$ in Sullivan (1984a). 
Reber, G. (1958). "Early radio astronomy at Wheaton, Illinois." Proc. IRE 46: 15-23. also reprinted in Sullivan (1984a).

Ryle, M. and Smith, F. G. (1948).* "A new intense source of radio-frequency radiation in the constellation of Cassiopeia." Nature 162: 462-3.

Ryle, M. and Vonberg, D. D. (1946).* "Solar radiation on 175 Mc./s." Nature 158: 339-40.

Ryle, M. and Vonberg, D. D. (1948). "An investigation of radio-frequency radiation from the sun." Proc. Roy. Soc. A193: 98-120.

Ryle, M., Smith, F. G. and Elsmore, B. (1950). "A preliminary survey of the radio stars in the Northern Hemisphere." Mon. Not. RAS 110: 508-23. erratum: Vol. 111, p. 641 (1951).

Ryle, M. (1952).* "A new radio interferometer and its application to the observation of weak radio stars." Proc. Roy. Soc. A211: 351-75.

Scheuer, P. A. G. (1984). "The development of aperture synthesis at Cambridge." pp. 249-65 in Sullivan (1984a).

Scheuer, P. A. G. (1990). "Radio source counts." pp. 331-45 in Modern Cosmology in Retrospect. (ed. R. Bertotti et al.). Cambridge: Cambridge Univ. Press.

Sullivan, W. T., III (1984a) (Ed.). The Early Years of Radio Astronomy: Reflections Fifty Years after Jansky. Cambridge: Cambridge University Press.

Sullivan, W. T., III (1984b). "Karl Jansky and the discovery of extraterrestrial radio waves." pp. $3-42$ in Sullivan (1984a).

Sullivan, W. T., III (1988). "Early years of Australian radio astronomy." pp. 308-44 in Australian Science in the Making. (ed. R. W. Home). Sydney: Cambridge University Press.

Sullivan, W. T., III (1990). "The entry of radio astronomy into cosmology: Radio stars and Martin Ryle's 2C survey." pp. 309-30 in Modern Cosmology in Retrospect. (ed. R. Bertotti et al.). Cambridge: Cambridge Univ. Press. 
Tom Landecker: Your picture showing the Cambridge 2C map showing radio sources in Galactic Co-ordinates reminds me of a persistent Cambridge habit. The galactic center was always moved from the center of the Aitoff projection. To a casual glance it appeared that a larger fraction of the sky had been observed than could actually be seen from Cambridge. The caption always said "The blank area is that part of the sky not visible from Cambridge". One result was to make the inaccessible Southern sky look less significant. Was this conscious?

W. T. Sullivan: The representation of $2 \mathrm{C}$ survey sources in the Aitoff equalarea projection seems fair enough to me. The survey covered -38 to +83 in declination, which is $63 \%$ of the sky. The missing southern portion was only $31 \%$. The map was centered on $\ell^{I}=0$, equivalent to $\ell^{I I}=32$.

U. Schwarz: Why did you not mention the influence of Reber on Oort and Dutch radioastronomy. Or do you consider non-continuum radioastronomy as not being radio astronomy but merely astronomy.

Sullivan: Given my allotted time and also the title of this colloquium, I decided to concentrate on radio source work and therefore omitted many topics such as the developments in Holland with the $21-\mathrm{cm}$ hydrogen line. As you say, it was indeed Reber's wartime papers in the Astrophysical Journal that first alerted Jan Oort to the possibilities of radio astronomy. 